\title{
Artigo 5
}

\section{Inovação Tecnológica e Polimorfismo de Fármacos}

\section{Carlito Lariucci, Hamilton B. Napolitano e Silvio Cunha}

O conhecimento do tipo de polimorfismo que um fármaco apresenta e das diferenças estruturais entre esses polimorfos é importante na pesquisa da indústria farmacêutica, pois a descoberta de um novo polimorfo, mesmo que seja metaestável, mas que possa ser produzido de forma controlada por uma empresa farmacêutica concorrente, constitui uma ameaça à patente de uma empresa sobre o seu princípio ativo. Nesse trabalho apresentase a importância dos metidos cristalográficos na análise de fármacos e suas aplicações na obtenção das informações estruturais precisas na identificação e caracterização de polimorfos, tais como: parâmetros da cela unitária, conformação molecular, empacotamento molecular e ligações de hidrogênio.

Palavras-chave: polimorfismo; cristalografia.

The knowledge of the polymorphism present in the specific drug and structural differences between these polymorphs are meaningful informations. The new polymorph discovered by another company yielded in controlled way becomes a kind of threat to the patent of active molecule. In this work we present the importance of crystallographic method to analyze the pharmaceutical molecules and its application aiming to obtain the most valuable structural information in the identification and characterization of the polymorphous: unit cell parameters, molecular conformation, molecular packing and hydrogen bonds.

Keywords: polymorphism; crystallography. 


\section{Introdução}

A inovação tecnológica é um componete principal no desenvolvimento da indústria famaceutica, e requer metas de produtividade e elevados níveis de investimento em Pesquisa, Desenvolvimento \& Inovação (PD\&I) ${ }^{1,2}$. Uma vez identificado um alvo biológico promissor envolvido em um estado de doença humana, o processo de descoberta de novas moléculas bioativas com potencial terapêutico poderá ser iniciado. Nesse momento, se dará a decisão por parte da companhia farmacêutica sobre o investimento de milhões de dólares neste longo processo, que passará, impreterivelmente, pelas fases complexas de descoberta e desenvolvimento. Um esquema geral das etapas envolvidas em PD\&I de fármacos é apresentado na Figura 1.

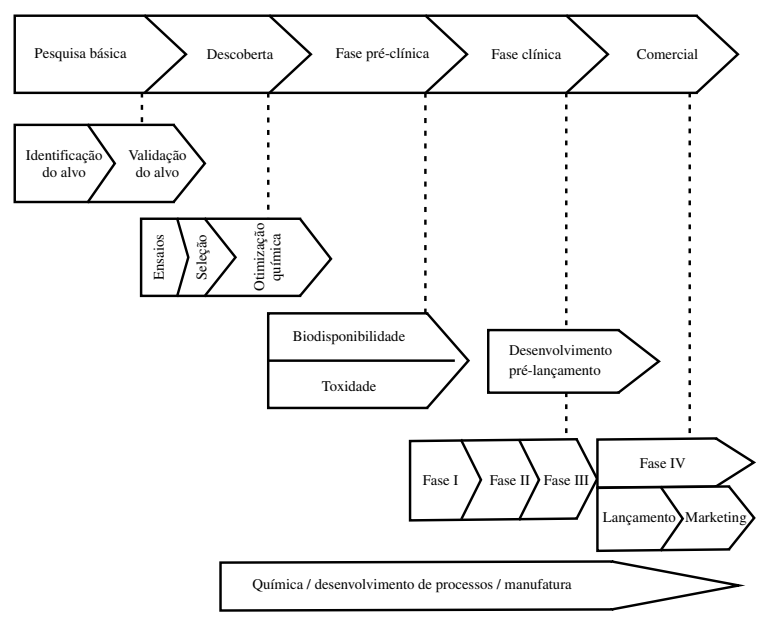

Figura 1: Etapas envolvidas no processo de PD\&I de fármacos.

O planejamento de novos agentes terapêuticos passa essencialmente pela capacidade de identificar moléculas pequenas capazes de interagir de forma seletiva à atividade do alvo macromolecular em um caminho que forneça eficácia e segurança no tratamento da doença de interesse. Fatores como toxidez, especificidade e o desafio de produzir compostos potentes e seletivos, fazem das proteínas o alvo da maioria dos fármacos.As principais companhias farmacêuticas têm investido em novas tecnologias para a descoberta de fármacos, procurando a integração das diversas áreas do conhecimento envolvidas neste processo de caráter multidisciplinar. Dentro deste complexo paradigma, destaca-se o crescimento do emprego de técnicas computacionais avançadas associadas ao tradicional trabalho experimental de laboratório. As plataformas para inovação são muitas e englobam diversos componentes das pesquisas clínicas em suas diferentes fases de desenvolvimento, associados a inúmeros outros componentes da pesquisa básica, que envolvem desde a validação de alvos moleculares até a descoberta e desenvolvimento de New Chemical Entities (NCEs)candidatas a novos fármacos ${ }^{1,2}$.

Os NCEs podem existir em diferentes formas com diferentes propriedades físicas e químicas e, quando encontrado no estado sólido, existem em mais de uma estrutura cristalina. O polimorfismo é uma propriedade importante e inclui todas as formas sólidas de uma mesma molécula (que apresentem a mesma fase de vapor, líquida ou em solução). O fenômeno do polimorfismo representa um desafio na indústria farmacêutica que pretende desenvolver drogas de qualidade consistente. Determinar a estrutura cristalina de um NCE é uma das primeiras etapas do desenvolvimento farmacêutico, e a verificação da existência de polimorfos deve ocorrer antes dos estudos clínicos e dos testes de estabilidade, pois a estrutura cristalina de uma droga possibilita (a) verificar quão facilmente ela pode ser formulada, (b) verificar a aplicação biológica e (c) informar a respeito de sua estabilidade. Diferenças na forma cristalina podem causar variações de propriedades físico-químicas dos compostos e, em conseqüência, diferenres formulações, biodisponibilidade e estabilidade.

O conhecimento dos polimorfos de um cristal é estratégico para a indústria farmacêutica, pois já houve casos de prejuízos em empresas depois de se observar mudanças nas formas polimorfas, que no final resultaram em qualidade inferior no teste de estabilidade do produto final, como, por exemplo, o medicamento Norvir $^{\mathbb{R}}$, de cápsulas semi-sólidas de Ritonavir. Em 1998, a Abbott Laboratories teve que reformular a droga antiHIV Ritonavir quando os processos de manufatura repentinamente começaram a produzir um polimorfo mais estável ${ }^{2}$. A descoberta de um novo polimorfo, mesmo que seja metaestável, mas que possa ser produzido de forma controlada por uma empresa farmacêutica concorrente, 
é uma ameaça à patente de uma empresa sobre o seu princípio ativo.

Uma vez descoberta uma nova droga, normalmente é a forma cristalina que é patenteada. Os polimorfos podem causar problemas, como: (a) um competidor poderia patentear e comercializar um polimorfo diferente; (b) um polimorfo indesejado (e potencialmente tóxico ou inerte) poderia ser manufaturado; (c) diferentes condições de manufatura ou condições de formulação podem produzir diferentes polimorfos; (d) é muito difícil determinar todos os possíveis polimorfos por experimentação; (e) podem ter propriedades inteiramente diferentes a partir da formulação desejada padrão, dentre outros.

Após a obtenção de uma nova formulação farmacêutica, várias perguntas precisam ser respondidas antes da comercialização, dentre elas ${ }^{3-5}$ : (1) Que tipo de polimorfismo exibe uma determinada droga? (2) Qual a diferença estrutural entre os polimorfos (empacotamento cristalino, ligações dehidrogênio, conformação molecular)? (3) De que maneira os polimorfos diferem em propriedades que podem afetar a qualidade e o desempenho de drogas (estabilidade, solubilidade, higroscopicidade, entre outros)? (4) As propriedades físicas podem ser previstas a partir da estrutura e vice-versa?

A Cristalografia de monocristais ${ }^{3,4}$, por difração de raios $X^{5,7}$, conforme ilustrado na Figura 2, fornece as mais precisas informações estruturais de compostos cristalinos, tais como: parâmetros da cela unitária; densidade; desordem cristalina; conformação molecular; empacotamento molecular; ligações de hidrogênio. O método cristalográfico fornece o conhecimento da disposição tridimensional dos átomos da estrutura molecular para compostos no estado cristalino. É uma metodologia interdisciplinar, com contribuições relevantes à Biologia Estrutural, Física, Química e a Tecnologia Farmacêutica.

O polimorfo pode ser identificado através da função matemática denominada densidade eletrônica $a^{6,7} \rho(\mathrm{r})$, construída através dos métodos cristalográficos, dado pela expressão

$$
\rho(\mathbf{r})=\frac{1}{V} \sum_{\mathbf{r}} F(\mathbf{h}) \exp i[-2 \pi \mathbf{h} \cdot \mathbf{r}]=\frac{1}{V} \sum_{i} \mid F(\mathbf{h}) \exp i[-2 \pi \mathbf{h} \cdot \mathbf{r}+\varphi(\mathbf{h})]
$$

onde $V$ é o volume da cela unitária e $\mathbf{F}(\mathbf{h})$ é o fator de estrutura na forma complexa, sendo seu módulo ao quadrado
$|\mathbf{F}(\mathbf{h})|^{2}$ proporcional à intensidade da reflexão medida para direção $\mathbf{h}$. A quantidade h•r corresponde ao produto escalar entre o vetor espalhamento no espaço recíproco $\mathbf{h}$ e o vetor posição no espaço direto $\mathbf{r}$. O conhecimento da função $\boldsymbol{\rho}(\mathbf{r})$ para cada posição $\mathbf{r}$ da cela unitária depende ainda do conhecimento das fases $\varphi(\mathbf{h})$. Uma vez conhecida a densidade eletrônica $\boldsymbol{\rho}(\mathbf{r})$, de forma plena se conhece de maneira unívoca a estrutura do polimorfo.

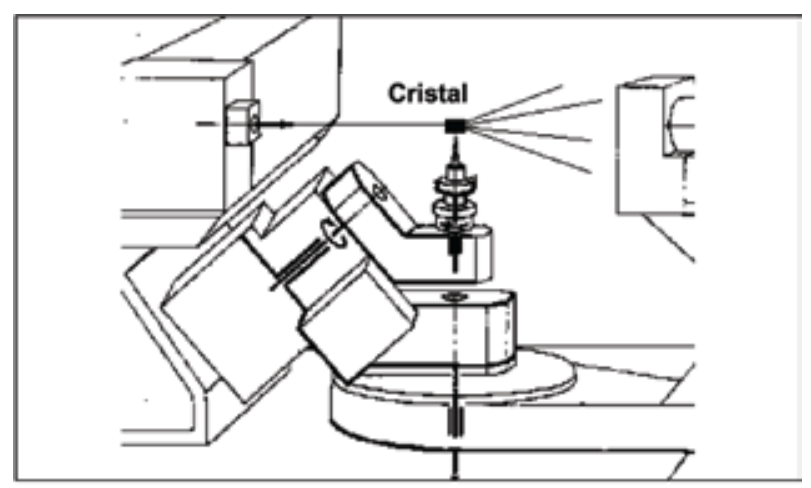

(a)

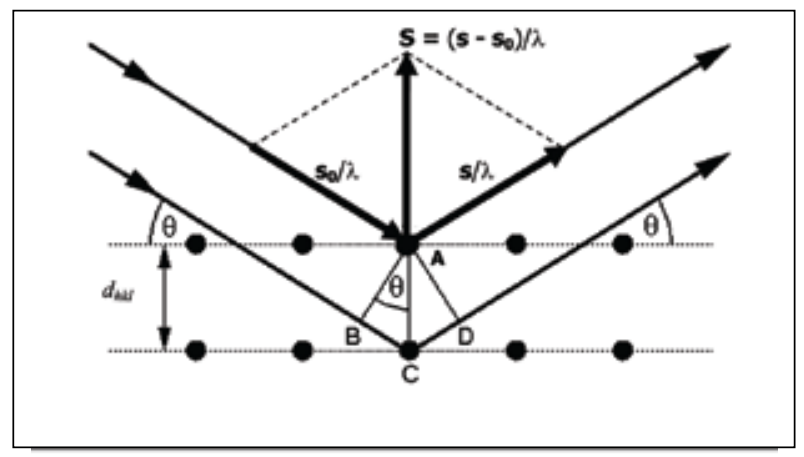

(b)

Figura 2: (a) Diagrama esquemático para difratômetros de monocristais, utilizados na coleta de dados de difração por raios X. (b) Representação esquemática (fora de escala) da equação de Bragg. $\theta$ é o ângulo entre o feixe de raios $\mathrm{X}$ incidente e o plano difrator $h k l$. A diferença de caminho entre as duas ondas espalhadas por A e C é $\mathrm{BC}+\mathrm{CD}=2 \mathrm{~d}_{h k l} \operatorname{sen} \theta$. A condição de difração é verificada quando a diferença de caminho for um múltiplo inteiro do comprimento de onda $\lambda$. O módulo do vetor $\mathrm{S}_{h k l}$ é o inverso da distância interplanar $\mathrm{d}_{h k l}$. 


\section{Importância do Polimorfismo na Indústria Farmacêutica}

Conforme descrito, um dos grandes problemas da indústria farmacêutica, no atual contexto de inovação e competitividade, é o polimorfismo ${ }^{8}$. O cerne do problema reside nas diferentes propriedades físicas e químicas de uma mesma substância em formas diferentes, tais como: densidade, índice de refração, ponto de fusão, condutividade elétrica ou térmica, higroscopicidade, energia livre e potencial químico, capacidade calorífica, pressão de vapor, solubilidade, estabilidade térmica, taxa de dissolução, cinética de reação do estado sólido, energia livre de superfície, hábito cristalino, cores, dureza, compressão, reatividade química e fotoquímica. Cabe destacar que todos esses parâmetros influenciam direta ou indiretamente nas pré-formulações farmacêuticas.

As condições de cristalização e produção das amostras podem levar ao surgimento de polimorfos em um grupo de cristais ou substâncias. O que se espera é obter cristais com forma e propriedade conhecidas ou então reproduzir os próprios resultados em laboratório. O mais importante, mas nem sempre alcançado, é conhecer o processo correto para obter os cristais com as características físicas, químicas e mecânicas desejadas, que são governadas por fatores termodinâmicos e cinéticos. Para se ter uma idéia da distinção entre a influência termodinâmica e cinética basta observar o clássico exemplo do carbono nas formas de grafite e diamante ${ }^{8}$. A forma cristalina do grafite é termodinamicamente preferida, mas fatores cinéticos, em particular a alta barreira de ativação, fazem com que a taxa de transformação do diamante para grafite seja infinitamente lenta. Contudo, o processo de cristalização pode ocorrer em posições de mínimos de energia metaestáveis ou estáveis. Uma mesma substância pode cristalizar nos dois mínimos de energia, com conformações diferentes em cada uma das posições. Assim teríamos um polimorfismo conformacional com diferença de energia entre eles variando de $1-2 \mathrm{kcal} / \mathrm{mol}^{8}$. Este é o caso da N-Benzoil-Guanidina?.

Outro tipo de polimorfismo muito comum é a incorporação de molécula(s) de solvente durante o processo de cristalização. Assim, pode-se afirmar que formas diferentes de cristais de uma substância podem possuir propriedades muito diferentes, além de se comportar como materiais diferentes. Estes conceitos têm implicações importantes nos campos da química associados com a produção e comercialização de moléculas na forma de materiais cristalinos (drogas, pigmentos, aditivo alimentar, explosivos, entre outros). A indústria precisa saber não somente a natureza exata do material no processo de produção e comercialização, mas também sua estabilidade com tempo, a variabilidade de suas propriedades físicas e químicas como uma função da forma cristalina. Em algumas áreas, por exemplo a indústria farmoquímica, a procura e caracterização de formas cristalinas do NCE se tornou um passo primordial para a escolha da melhor forma para formulação, produção, estabilidade e para proteção da propriedade intelectual ${ }^{2,8}$.

\section{Polimorfos de Potenciais Fármacos}

Sistemas polimórficos proporcionam oportunidade únicas para estudar a competição entre forças intermoleculares e correlações entre mudanças na estrutura molecular e o empacotamento cristalino. A síntese de guanidinas é um tema de intensa investigação pelo fato desta classe de composto está presente em uma variedade de substâncias com atividade biológica, tanto naturais quanto sintéticas. A elucidação estrutural tridimensional de guanidinas fornece informações sobre pontes de hidrogênio intra e intermoleculares, que podem ser úteis na compreensão da atividade biológica desta classe de compostos. Descrevemos aqui o polimorfismos da N-Benzoil-Guanidina: 4-Metoxi-1-[4metoxianilino(fenilcarbonilimino)metilamino]benzeno", um potencial NCE.

O trabalho de determinação da estrutura tridimensional de ambos polimorfos foi obtido através da cristalografia de raios $\mathrm{X}$, e envolveu as seguintes etapas ${ }^{10-15}$, (1) coleta de dados, (2) processamento dos dados, (3) resolução da estrutura, (4) refinamento e (5) validação e análise do modelo cristalográfico.

O polimorfo (I) é caracterizado estruturalmente através dos parâmetros cristalográficos, como cela unitária monoclínica ( $a=11,70 \AA ; b=9,16 \AA ; c=18,67$ 
$\AA ; \alpha=\gamma=90^{\circ}$ e $\beta=96,26^{\circ}$ ), grupo espacial equivalente a simetria $\mathrm{P} 2 / \mathrm{n}$ com quatro moléculas dentro da cela unitária $(Z=4)$. A descrição estrutural do forma 1 pode ser vista na Figura 3. O polimorfo (II) é caracterizado estruturalmente através dos parâmetros cristalográficos, como cela unitária monoclínica $(a=8.13 \AA ; b=8.55 \AA$; $c=14,76 \AA ; \alpha=97,52^{\circ}, \beta=94,02^{\circ}$ e $\gamma=100,04^{\circ}$ ), grupo espacial equivalente a simetria P-1 com apenas duas
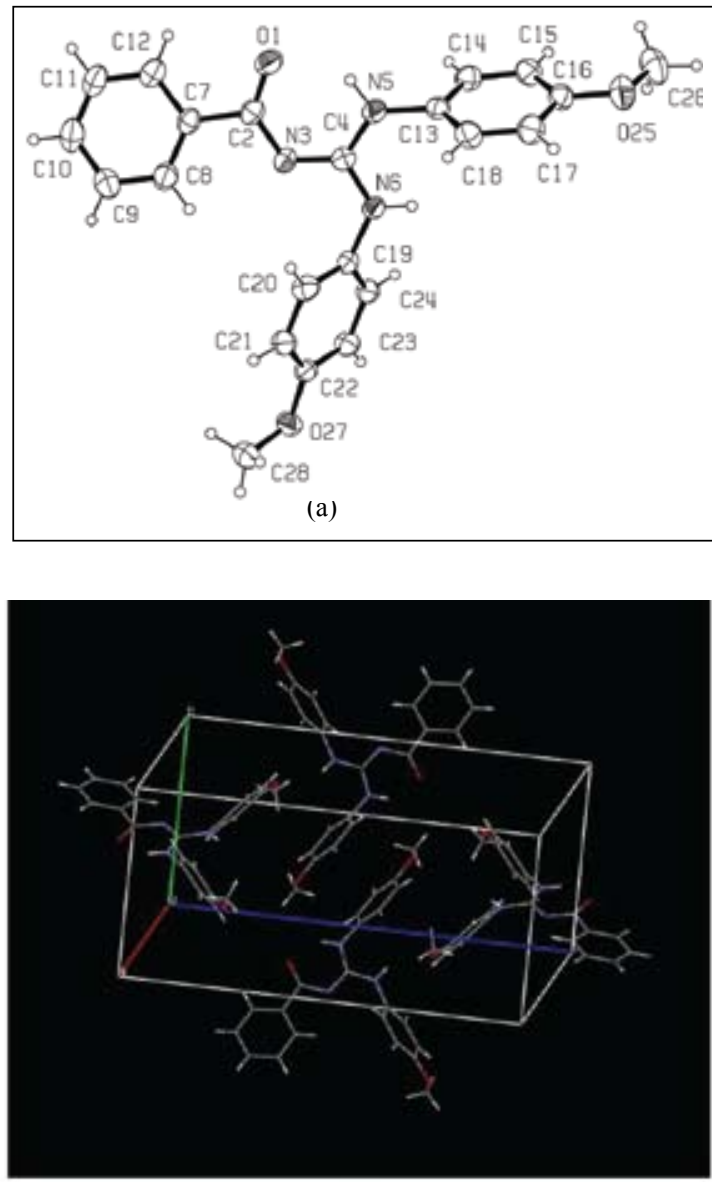

(b)

Figura 3: (a) Representação da estrutura tridimensional molecular do polimorfo I. (b) Representação da estrutura cristalina do polimorfo I ilustrando a simetria do grupo espacial $\mathrm{P} 2 \mathrm{1} / \mathrm{n}$. moléculas dentro da cela unitária $(Z=2)$. A descrição estrutural do forma (II) pode ser vista na Figura 4. Os diferentes arranjos espaciais observados para a acomodação molecular entre os polimorfos I e II implicam em diferentes padrões de interações não-covalentes e, portanto, em diferentes propriedades físico-químicas. A Figura 5 ilustra as diferenças estruturais moleculares entre os polimorfos I e II.
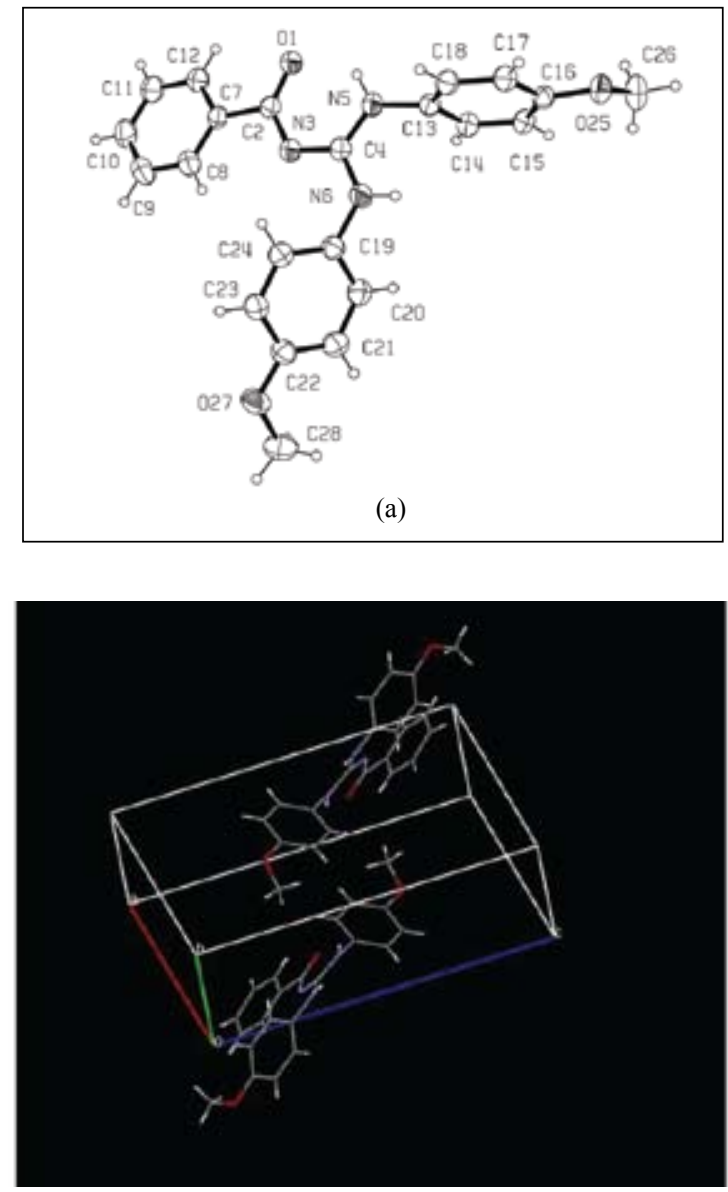

(b)

Figura 4: (a) Representação da estrutura tridimensional molecular do polimorfo II. (b) Representação da estrutura cristalina do polimorfo II ilustrando a simetria do grupo espacial P-1. 


\section{Artigo 5}

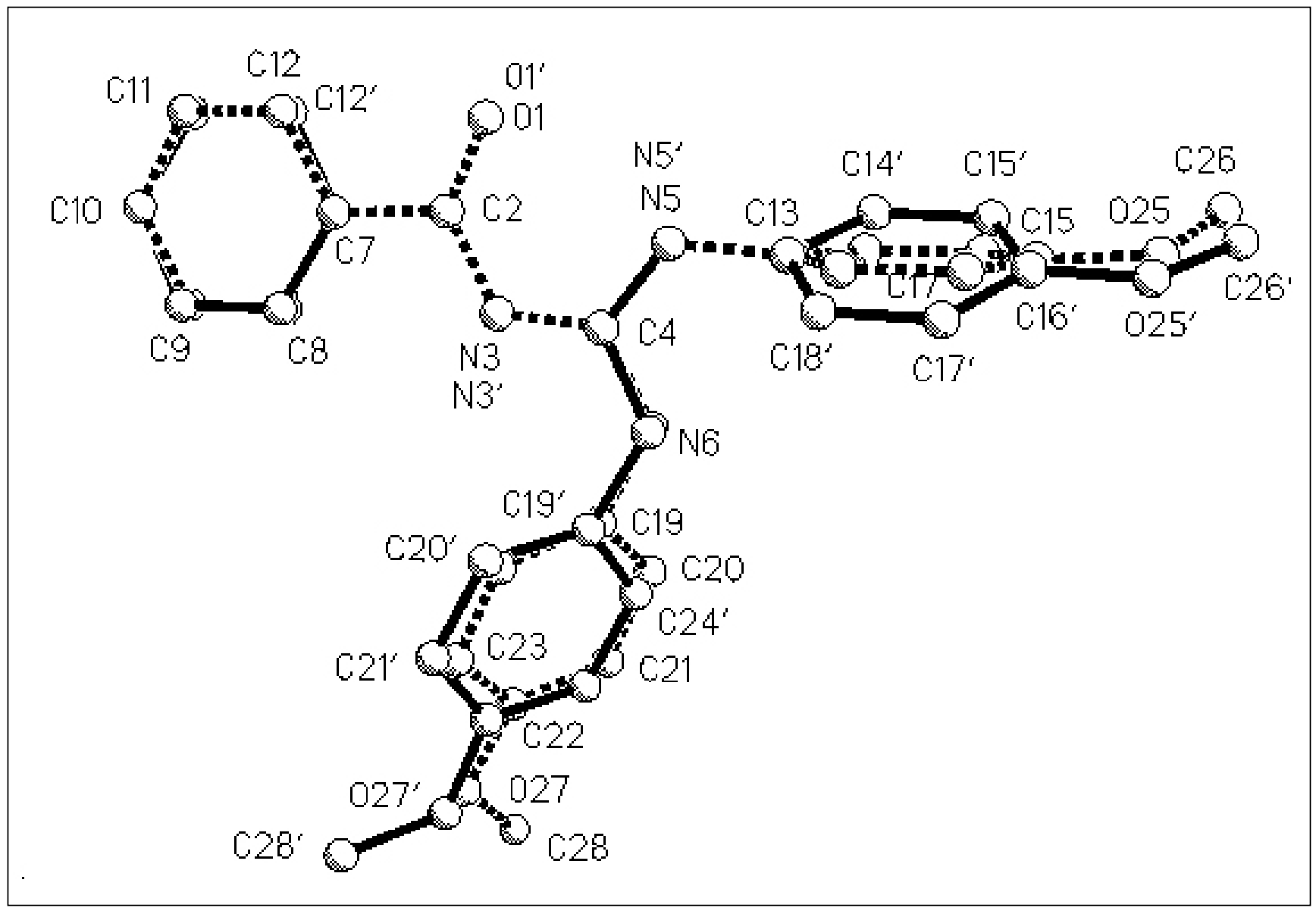

Figura 5: Representação das diferenças estruturais da estrutura tridimensional molecular entre os polimorfos I e II.

\section{Considerações Finais}

$\mathrm{O}$ estudo do polimorfismo vem, nas últimas décadas, ganhando grande importância para a indústria farmacêutica ${ }^{8,16}$. A necessidade de seu estudo, sobretudo na pesquisa por novos fármacos e no estudo de préformulações, fica evidente pelo exemplo discutido. Qualquer NCE pode possuir polimorfismo e não existe uma boa relação a priori entre (1) a classe da substância química e (2) a existência de polimorfos. Para tanto, existem diversas técnicas de análise e de manipulação da forma cristalina que tornam possível a obtenção da isoforma que melhor atenda à determinada formulação. A metodologia mais versátil e que se destaca nesta tarefa é a cristalografia de raios $\mathrm{X}$.

\section{REFERÊNCIAS}

1. Yu, L.; Reutzel, S. M. \& Stepherson, G. A. PSTT, v. 1(3), p. 118, 1998.

2. Snider, D. A; Addicks, W \& Owens, $W$. Advanced Drug Delivery Reviews, v. 56, p. 391, 2004.

3. Stout, G. \& Jensen, L. H. X-ray structure determination - A practical guide, 2a. ed.; The Macmillan Company: London, 1989.

4. Viterbo, D. Em Fundamentals of Crystallography; 2a. ed.; IUCr e Oxford Science Publication: New York, 2002. 
5. Cullity, B. D. Elements of X-ray diffraction, 2a. ed.; Addison-Wesley Publishing Company, Inc: Massachusetts, 1978.

6. Giacovazzo, C. Em Fundamentals of Crystallography; IUCr e Oxford Science Publication: New York, 1992.

7. Giacovazzo, C. Fundamentals of Crystallography, 2a. ed.; IUCr e Oxford Science Publication: New York, 2002.

8. Bernstein, J. Polymorphism in Molecular Crystals. Oxford University Press. UK, 2007; Bernstein, J. Appl. Phys. 26 B66-B76, 1993.

9. Cunha, S., Lariucci, C \& Napolitano, H. B. Artigo em preparação da N-Benzoil-Guanidina: 4-Metoxi1-[4-metoxianilino(fenilcarbonilimino)metilamino] benzeno, incluindo a descrição da Forma I e II. 2008.

10. Altomare, A.; Burla, M. C.; Camalli, M.; Cascarano, G. L.; Giacovazzo, C.; Guagliardi, A.; Moliterni, A. G. G.; Polidori, G. \& Spagna, R. J. Appl. Cryst., v.32, p.115, 1999.

11. Sheldrick, G. M. SHELXL-97 Program for the Refinement of Crystal Structures. University of Göttingen. Germany, 1997.

12. Sheldrick, G. M.; SHELXS-97 Program for the Solution of Crystal Structures. University of Göttingen. Germany, 1990.
13. Beurskens, P. T.; Beurskens, G.; Gelder, R.; Garcia-Granda, S.; Gould, R. O.; Israel, R. \& Smits, J. M. M. The DIRDIF-99 Program System. Crystallography Laboratory, University of Nijmegen, The Netherlands, 1999.

14. CSD (Cambridge Structural Database). Cambridge Crystallographic Data Centre. 12 Union Road, Cambridge. England, 2000.

15. Le Page, Y. J. Appl. Cryst., v. 21, p. 983, 1988.

16. Singhal, D \& Curatolo, W. Advanced Drug Delivery Reviews, v. 56, p. 335, 2004.

\section{Carlito Lariucci*1, Hamilton B. Napolitano ${ }^{2}$ e Silvio Cunha $^{3}$}

\author{
${ }^{1}$ Instituto de Física, UFG. CP 131, 74001-970, Goiânia, GO. \\ ${ }^{2}$ Ciências Exatas \& Tecnológicas, UEG. BR 153, Km 98, 75133-050 \\ Anápolis, GO. \\ ${ }^{3}$ Instituto de Química, UFBA. Campus de Ondina, 40170-290 \\ Salvador, BA. \\ *E-mail: lariucci@if.ufg.br
}

